\title{
Conversion of Percutaneous Cholecystostomy to Endoscopic Gallbladder Stenting by Using the Rendezvous Technique
}

\author{
Kwangwoo Nam and Jun-Ho Choi \\ Division of Gastroenterology, Department of Internal Medicine, Dankook University Hospital, Dankook University College of Medicine,
} Cheonan, Korea

We report the successful conversion of percutaneous cholecystostomy (PC) to endoscopic transpapillary gallbladder stenting (ETGS) with insertion of an antegrade guidewire into the duodenum. An 84-year-old man presented with severe acute cholecystitis and septic shock. He had significant comorbidities, and emergent PC was successfully performed. Subsequent ETGS was attempted but unsuccessful owing to difficulties with cystic duct cannulation. However, via the PC tract, the guidewire was passed antegradely into the duodenum, and ETGS with a double-pigtail plastic stent was successfully performed with the rendezvous technique. The PC tube was removed, and no recurrence was reported during the 17-month follow-up period. Conversion of PC to ETGS is a viable option in patients with acute cholecystitis who are not candidates for surgery. Antegrade guidewire insertion via the PC tract may increase the success rate of conversion and decrease the risk of procedure-related complications. Clin Endosc 2017;50:301-304

Key Words: Cholecystitis, acute; Cholecystostomy; Drainage; Cholangiopancreatography, endoscopic retrograde

\section{INTRODUCTION}

The treatment of choice for acute cholecystitis is laparoscopic cholecystectomy; however, this procedure is unsuitable for patients who are critically ill and have significant comorbidities. ${ }^{1}$ In such patients, percutaneous cholecystostomy (PC) is an effective alternative option, as it is a safe and minimally invasive radiologic procedure with which immediate gallbladder decompression and prompt clinical improvement are possible. The technical success rate and clinical response rate associated with PC has been reported to be up to $100 \%$ and 95\%, respectively. ${ }^{2-4}$ However, long-term placement of a PC tube has several disadvantages, such as patient discomfort, the

Received: August 17, 2016 Revised: September 27, 2016

Accepted: September 28, 2016

Correspondence: Jun-Ho Choi

Division of Gastroenterology, Department of Internal Medicine, Dankook University Hospital, Dankook University College of Medicine, 201 Manghyang-ro, Dongnam-gu, Cheonan 31116, Korea

Tel: +82-41-550-3098, Fax: +82-41-556-3256, E-mail: mdcjh78@gmail.com

(cc) This is an Open Access article distributed under the terms of the Creative Commons Attribution Non-Commercial License (http://creativecommons.org/ licenses/by-nc/3.0) which permits unrestricted non-commercial use, distribution, and reproduction in any medium, provided the original work is properly cited. need for repeated procedures, and catheter dislodgement. ${ }^{4,5}$ In addition, the recurrence rate of cholecystitis after removal of the PC tube has been reported to be $33 \%-41 \%$ in previous studies. ${ }^{2}$

Endoscopic transpapillary gallbladder stenting (ETGS) is another alternative option. With this procedure, internal drainage can be performed and the patient's quality of life can be preserved. ETGS requires biliary and cystic duct cannulation. During ETGS, the guidewire is advanced retrogradely into the gallbladder, which is the most challenging part of the procedure. However, in patients who have already undergone PC, the guidewire can be advanced antegradely through the gallbladder into the duodenum, which improves the success rate of cystic duct cannulation. Herein, we report the case of a patient who initially underwent PC for severe acute cholecystitis and, after recovery, underwent successful conversion to ETGS with the rendezvous technique.

\section{CASE REPORT}

An 84-year-old man with diabetes and severe coronary 


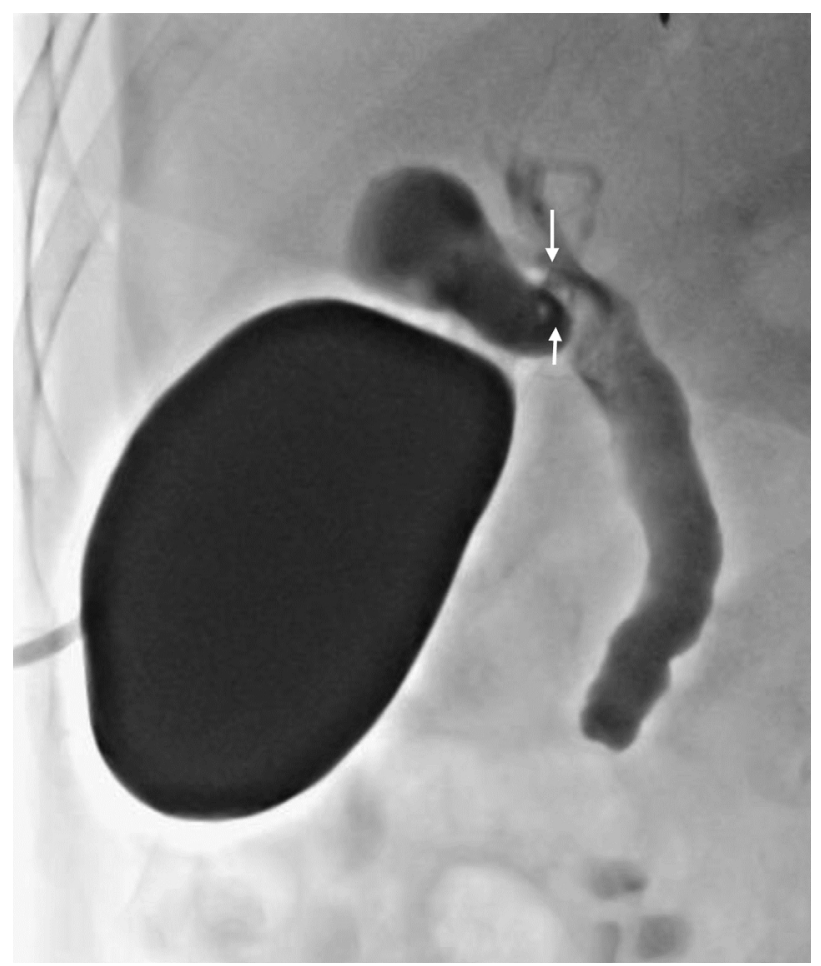

Fig. 1. Fluoroscopic image showing a stricture in the cystic duct (arrows).

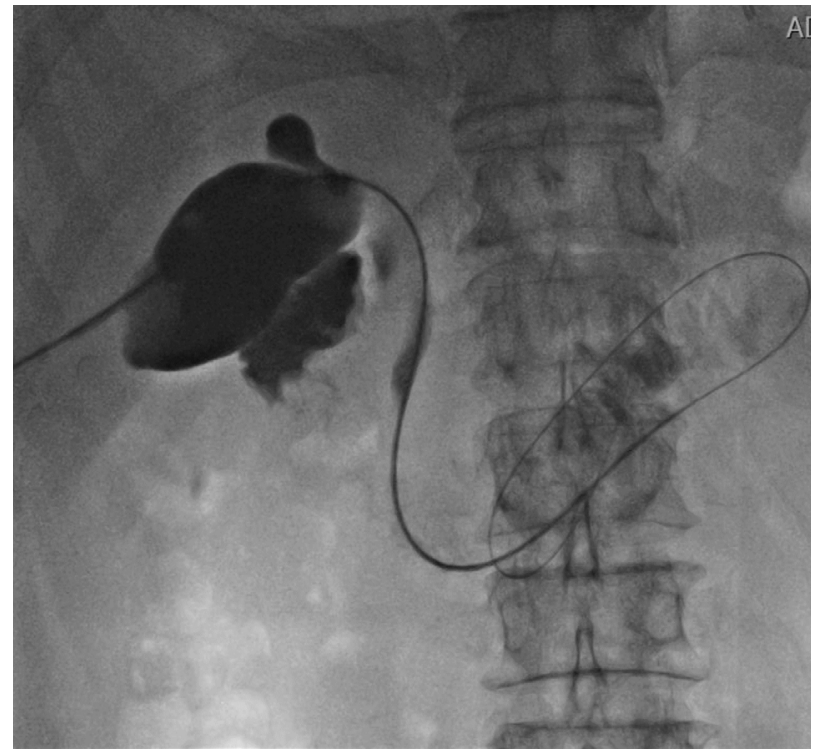

Fig. 2. Fluoroscopic image showing a percutaneous transgallbladder guidewire being advanced antegradely through the gallbladder and common bile duct into the duodenum.

artery disease came to our hospital with a complaint of right upper-quadrant pain. He developed severe acute cholecystitis complicated by sepsis, and hemodynamic instability. He underwent emergent PC, which resulted in clinical improvement. After his condition improved, we considered removing the PC tube. However, abdominal pain recurred immediately

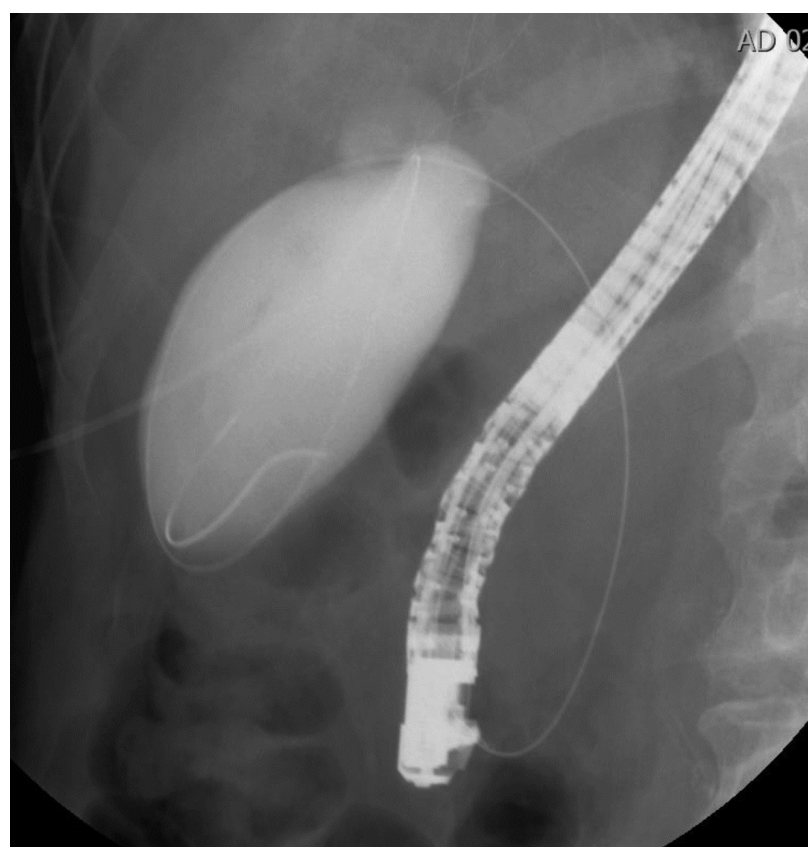

Fig. 3. Fluoroscopic image of coiling of the guidewire within the lumen of the gallbladder.

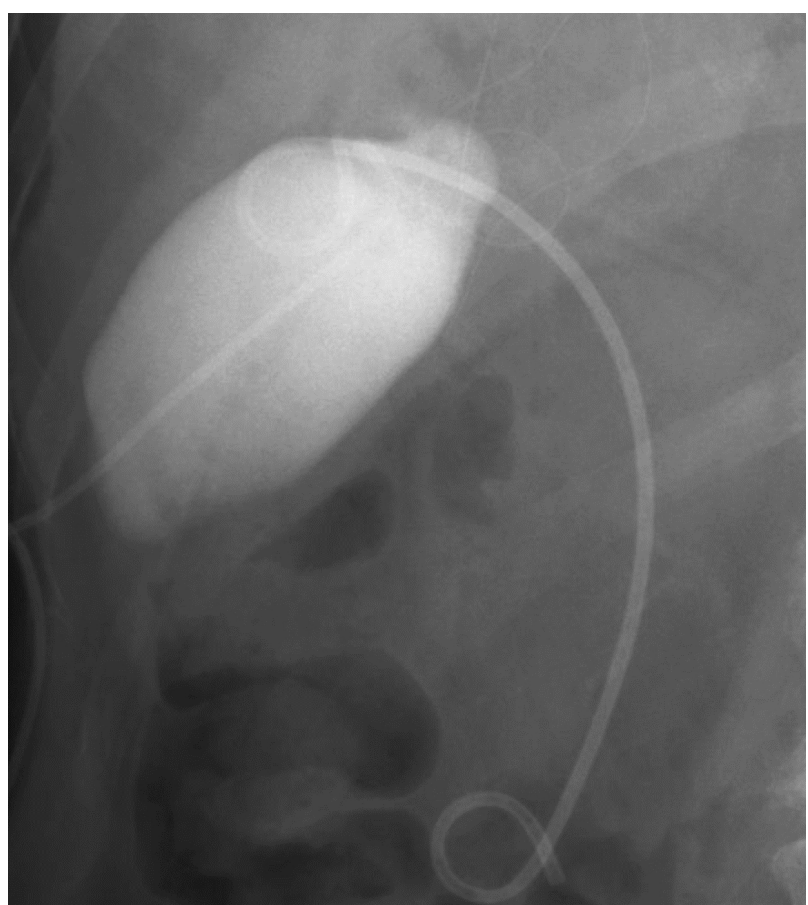

Fig. 4. Fluoroscopic image after positioning of the transpapillary gallbladder stent.

after the PC tube was clamped. Given the high surgical risk associated with his condition, endoscopic retrograde cholangiopancreatography for ETGS was performed 1 week later. Selective cannulation of the cystic duct was attempted; however, it was unsuccessful, even after guidewires of various sizes and papillotomes such as Autotome (Boston Scientific, 
Microvasive, Marlboro, MA, USA), were used, because of cystic duct stricture and tortuosity (Fig. 1). To facilitate cystic duct access, the cholecystostomy tube was replaced with a hydrophilic guidewire that was passed antegradely into the duodenum (Fig. 2). Then, a duodenoscope (JF-260V; Olympus Medical Co., Tokyo, Japan) was inserted up to the papilla, at the point where the guidewire was coming out. The guidewire was grasped with forceps and pulled out through the accessory channel of the duodenoscope. Cystic duct cannulation was achieved with a cannula placed over the antegradely placed guidewire (Fig. 3). Finally, a 7-F, 12-cm-long double-pigtail plastic stent (Cook Medical, Bloomington, IN, USA) was advanced over the wire with one loop left in the gallbladder and one in the duodenum (Fig. 4). The percutaneous catheter was removed after the procedure. Cholecystitis did not recur, and the patient's condition remained stable during the 17-month follow-up.

\section{DISCUSSION}

Selective deep cannulation of the cystic duct is essential for a successful ETGS. However, even the most experienced endoscopists fail in cannulating the cystic duct in $21 \%$ of cases. ${ }^{6}$ When a selective cystic duct cannulation fails, PC allows successful immediate gallbladder drainage and retrograde access for a subsequent rendezvous technique. ${ }^{7-11}$ This combined technique increases the success rate of cystic duct cannulation and facilitates the placement of a gallbladder stent. We report the case of a patient who had initially undergone emergent PC and, after clinical improvement, underwent successful conversion to ETGS with the rendezvous technique. The guidewire was anterogradely placed through the PC tract to the duodenum, and a double-pigtail plastic stent was retrogradely inserted by using the transpapillary approach. The patient had no recurrent cholecystitis during the 17 -month follow-up period.

In patients who develop severe acute cholecystitis but are medically unfit for cholecystectomy because of significant comorbidities, emergent PC is the treatment of choice. After clinical improvement, however, long-term placement of the PC tube is troublesome. Furthermore, simple removal of the PC tube may lead to frequent recurrence of cholecystitis. ${ }^{2}$ In this situation, ETGS after PC should be considered. Because transmural puncture is not performed during the procedure, the risk of bile leakage, bile peritonitis, and bleeding is low. ${ }^{12}$ ETGS can be used as a bridge therapy (if elective cholecystectomy is scheduled) or as a definite therapy (if there is a contraindication to elective cholecystectomy). ${ }^{6,71,1,14}$ A double-pigtail plastic stent can preserve cystic duct patency and prevent recurrent cholecystitis. After stent placement, a "wait-and-see" approach is recommended, because continuous bile drainage is possible along the occluded stent (the "wicking phenomenon"). ${ }^{6.15}$ In a long-term follow-up study, a mean stent patency of 760 days was reported without regular stent change. ${ }^{6}$

The main drawback of ETGS is its lower success rate than that of PC. The technical success rate and clinical response rate have been reported to be $96 \%$ and $88 \%$, respectively, which are lower than those of PC. This is mainly due to the difficulty associated with cystic duct cannulation. In cystic duct cannulation, the guidewire maneuver is difficult because of the corkscrew shape and narrowness of the cystic duct, as well as the presence of the spiral valve of Heister. In addition, the cystic duct may show stricture or obstruction in acute cholecystitis. ${ }^{7}$ In the present case, cystic duct stricture and tortuosity were also found in the cholecystogram (Fig. 1), which were associated with failure of cystic duct cannulation through the transpapillary approach. However, by using the PC tract, antegrade guidewire insertion into the duodenum was more easily performed because there was enough space for guidewire manipulation. After the successful ETGS placement, the PC tube was removed and there was no bile leak after tube removal.

Several cases of percutaneous transgallbladder rendezvous for ETGS placement have been reported. Elmunzer et al. reported on 2 patients who underwent PC for severe acute cholecystitis and, after recovery, underwent ETGS with the guidewire advanced through the cholecystostomy tract into the duodenum. ${ }^{15}$ During the long-term follow-up period (10 and 6 months of follow-up), the patients remained stable. Maekawa et al. reported on 5 patients who underwent ETGS by passing the guidewire antegradely into the duodenum via the PC route and then by using the guidewire retrogradely. The success rate of the procedure was $100 \%$ (5 of 5) and was higher than that of emergent ETGS (77.5\%, 31 of 40). Hatanaka et al. reported on 2 patients with unresectable cancer who had undergone PC because of failed ETGS, and then underwent a successful ETGS with a rendezvous approach. ${ }^{8}$ During the rendezvous procedure, a PC-placed guidewire was grasped at the tip of the duodenoscope; then, the duodenoscope and wire were withdrawn. In case of difficulty in grasping the wire with forceps, Matsubayashi et al. suggested a parallel cannulation technique along with PC rendezvous. ${ }^{9}$ A modified rendezvous technique in which the guidewire was retrogradely passed into the gallbladder after end-to-end contact between the tips of the sphincterotome and the PC catheter at the orifice of the ampulla may also be a feasible option. ${ }^{16}$ However, extending a PC tube into the duodenal lumen is often difficult.

In conclusion, conversion of PC to ETGS is a viable option in patients with acute cholecystitis who are unfit for cholecys- 
tectomy. Antegrade guidewire insertion via the PC tract and subsequent ETGS placement by using the rendezvous technique may increase the success rate and decrease the risk of procedure-related complications. However, further studies are needed to evaluate the feasibility of conversion with antegrade guidewire insertion in patients with acute cholecystitis and severe comorbidities.

Conflicts of Interest

The authors have no financial conflicts of interest.

\section{REFERENCES}

1. Spira RM, Nissan A, Zamir O, Cohen T, Fields SI, Freund HR. Percutaneous transhepatic cholecystostomy and delayed laparoscopic cholecystectomy in critically ill patients with acute calculus cholecystitis. Am J Surg 2002;183:62-66.

2. McKay A, Abulfaraj M, Lipschitz J. Short- and long-term outcomes following percutaneous cholecystostomy for acute cholecystitis in highrisk patients. Surg Endosc 2012;26:1343-1351.

3. Akhan O, Akinci D, Ozmen MN. Percutaneous cholecystostomy. Eur J Radiol 2002;43:229-236.

4. Itoi $\mathrm{T}$, Coelho-Prabhu N, Baron TH. Endoscopic gallbladder drainage for management of acute cholecystitis. Gastrointest Endosc 2010;71:1038-1045

5. Jang JW, Lee SS, Song TJ, et al. Endoscopic ultrasound-guided transmural and percutaneous transhepatic gallbladder drainage are comparable for acute cholecystitis. Gastroenterology 2012;142:805-811.

6. Lee TH, Park DH, Lee SS, et al. Outcomes of endoscopic transpapillary gallbladder stenting for symptomatic gallbladder diseases: a multicenter prospective follow-up study. Endoscopy 2011;43:702-708.

7. Maekawa S, Nomura R, Murase T, Ann Y, Oeholm M, Harada M. Endoscopic gallbladder stenting for acute cholecystitis: a retrospective study of 46 elderly patients aged 65 years or older. BMC Gastroenterol 2013;13:65.

8. Hatanaka T, Itoi T, Ijima M, et al. Efficacy and Safety of Endoscopic Gallbladder Stenting for Acute Cholecystitis in Patients with Concomitant Unresectable Cancer. Intern Med 2016;55:1411-1417.

9. Matsubayashi H, Maeda A, Fukutomi A, Ono H. A case of choledocholithiasis treated by parallel cannulation along with PTGBD rendezvous. Clin J Gastroenterol 2009;2:36-38.

10. Banerjee B, Harshfield DL, Teplick SK. Percutaneous transcholecystic approach to the rendezvous procedure when transhepatic access fails. J Vasc Interv Radiol 1994;5:895-898.

11. Okuno M, Iwashita T, Yasuda I, et al. Percutaneous transgallbladder rendezvous for enteroscopic management of choledocholithiasis in patients with surgically altered anatomy. Scand J Gastroenterol 2013;48:974-978.

12. Law R, Grimm IS, Stavas JM, Baron TH. Conversion of Percutaneous Cholecystostomy to Internal Transmural Gallbladder Drainage Using an Endoscopic Ultrasound-Guided, Lumen-Apposing Metal Stent. Clin Gastroenterol Hepatol 2016;14:476-480.

13. Schlenker C, Trotter JF, Shah RJ, et al. Endoscopic gallbladder stent placement for treatment of symptomatic cholelithiasis in patients with end-stage liver disease. Am J Gastroenterol 2006;101:278-283.

14. Mutignani M, Iacopini F, Perri V, et al. Endoscopic gallbladder drainage for acute cholecystitis: technical and clinical results. Endoscopy 2009;41:539-546.

15. Elmunzer BJ, Novelli PM, Taylor JR, Piraka CR, Shields JJ. Percutaneous cholecystostomy as a bridge to definitive endoscopic gallbladder stent placement. Clin Gastroenterol Hepatol 2011;9:18-20.

16. Lee TH, Park SH, Lee SH, et al. Modified rendezvous intrahepatic bile duct cannulation technique to pass a PTBD catheter in ERCP. World J Gastroenterol 2010;16:5388-5390. 